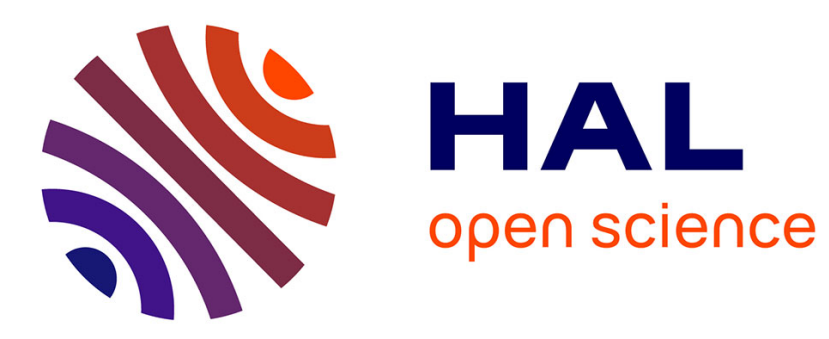

\title{
Control of VTOL Vehicles with Thrust-Tilting Augmentation
}

Minh-Duc Hua, Tarek Hamel, Claude Samson

\section{To cite this version:}

Minh-Duc Hua, Tarek Hamel, Claude Samson. Control of VTOL Vehicles with Thrust-Tilting Augmentation. 19th World Congress. The International Federation of Automatic Control., Aug 2014, Cape Town, South Africa. pp.2237 - 2244, 10.3182/20140824-6-ZA-1003.00097 . hal-01377793

\section{HAL Id: hal-01377793 \\ https://hal.science/hal-01377793}

Submitted on 7 Oct 2016

HAL is a multi-disciplinary open access archive for the deposit and dissemination of scientific research documents, whether they are published or not. The documents may come from teaching and research institutions in France or abroad, or from public or private research centers.
L'archive ouverte pluridisciplinaire HAL, est destinée au dépôt et à la diffusion de documents scientifiques de niveau recherche, publiés ou non, émanant des établissements d'enseignement et de recherche français ou étrangers, des laboratoires publics ou privés. 


\title{
Control of VTOL Vehicles with Thrust-Tilting Augmentation
}

\author{
Minh-Duc Hua * Tarek Hamel ${ }^{* *}$ Claude Samson *** \\ * ISIR UPMC-CNRS, Paris, France (e-mail: hua@isir.upmc.fr) \\ ** I3S UNSA-CRNS, Sophia-Antipolis, France \\ (e-mail: thamel@i3s.unice.fr) \\ *** INRIA and I3S UNSA-CRNS, Sophia-Antipolis, France \\ (e-mails: claude.samson@inria.fr, csamson@i3s.unice.fr)
}

\begin{abstract}
An approach to the control of a VTOL vehicle equipped with complementary thrusttilting capabilities that nominally yield full actuation of the vehicle's position and attitude is developed. The particularity and difficulty of the control problem are epitomized by the existence of a maximum tilting angle which forbids complete and decoupled control of the vehicle's position and attitude in all situations. This problem is here addressed via the formalism of primary and secondary objectives and by extending a solution previously derived in the fixed thrust-direction case. The proposed control design is also illustrated by simulation results involving a quadrotor UAV with all propellers axes pointing in the same monitored tilted direction.
\end{abstract}

\section{INTRODUCTION}

Mechanical design and feedback control of small aerial vehicles possessing thrust vectoring capabilities have received an increasing interest in recent years and given rise to various declinations (Cetinsoy et al. [2012], Kendoul et al. [2005], Naldi et al. [2008], Notarstefano and Hauser [2010], Papachristos et al. [2011], Pflimlin et al. [2010], Russo et al. [2011], Ryll et al. [2012]). For instance, the concept of twin tilt-rotors (Kendoul et al. [2005], Papachristos et al. [2011]) is well exemplified by the Bell Eagle Eye and the Bell Boeing V-22 Osprey. Other concepts such as three-rotors UAVs with one tilting rotor (SalazarCruz et al. [2008]), tilt-wing UAVs (Cetinsoy et al. [2012]), and quadrotor UAVs with two orthogonal tilting axes (Ryll et al. [2012]) are also worth mentioning.

Thrust vectoring for an aerial vehicle is the ability to modify the direction of the propulsion thrust with respect to (w.r.t.) a body-fixed frame. This feature can be used either for attitude (i.e., orientation) control, when the thrust rotation center is located at some distance of the vehicle's center of mass (CoM) and thrust vectoring yields torque creation, as in the case of rocket nozzle tilting or ducted-fan airflow derivation via the use of rotating surfaces (Naldi et al. [2008], Pflimlin et al. [2010]), or for attitude/position control decoupling, when the thrust rotation center is near the CoM and complementary actuation for attitude control is available, as in the case of $\mathrm{V} /$ STOL aircraft whose fuselage orientation is controlled independently of the vehicle's longitudinal motion (Notarstefano and Hauser [2010], Russo et al. [2011]). As a matter of fact, thrust vectoring can also be used to achieve a combination of the aforementioned objectives. This multiple usage renders the term thrust-vectoring somewhat imprecise. We use here the term thrust-tilting in reference

\footnotetext{
^ This work was supported by the French Agence Nationale de la Recherche through the ANR ASTRID SCAR project "Sensory Control of Aerial Robots" (ANR-12-ASTR-0033).
}

to the second possibility, i.e. attitude and longitudinal motion control decoupling. In this case, thrust direction tilting involves two actuated degrees of freedom (d.o.f.) which complement the conventional four actuated d.o.f. associated with common aerial vehicles, namely thrust intensity plus three torque components necessary for complete attitude control. This yields six independent actuated d.o.f. that allow for the complete control of the six dimensional state associated with the position and attitude of the vehicle's body. A similar objective is addressed in Ryll et al. [2012] where the vehicle under consideration is a quadrotor UAV whose propellers axes rotate two by two about one of the two orthogonal axes of the quadrotor's "cross" structure supporting the propellers. This configuration thus involves four additional motors (one for each propeller) but yields, at the vehicle's body level, only two additional independently actuated d.o.f. as a result of inevitable actuation coupling when more than six independent actuators are employed. The control design proposed in Ryll et al. [2012] basically relies on exact linearization of the vehicle's motion equations. This control strategy, combined with actuation redundancy, in turn leads to a control calculation based on the use of pseudo-inverse matrices and on solving a complementary optimization problem (energy expenditure minimization, for example, as proposed in the paper). However, thrusttilting angle limitations are not taken into account in this study.

With respect to Ryll et al. [2012], we here address thrust tilting in the form of a generic problem whose solution potentially applies to a large panel of aerial vehicles with extended flight envelopes, including the omnicopter considered in Ryll et al. [2012] and V/STOL aircraft. Such a claim of generality imposes to take aerodynamic forces acting on the vehicle into account. For control design purposes, simplified models of these forces are used. Another difference with Ryll et al. [2012] is that a non-redundant thrust tilting actuation -i.e. involving only the two d.o.f. 
associated with the modification of an axis direction in the three-dimensional Euclidean space- is considered. The proposed (nonlinear) control design is also different. It is in fact an extension of the one presented in Hua et al. [2009], Hua et al. [2013] for reference velocity or position tracking in the case where the thrust direction is fixed, and it is based on a Lyapunov-like approach. An important original outcome of the present study is that vectoring limitations are taken into account explicitly. More precisely, it is assumed that the thrust tilting angle with respect to a "neutral" direction, corresponding for instance to the one associated with the fixed direction of a conventional quadrotor UAV, cannot exceed a known threshold. Due to this limitation, independent control of the vehicle's attitude and position is no longer always possible. This leads naturally to set priorities between complementary control objectives. Accordingly, the proposed control methodology involves a primary objective associated with the reference velocity or position asymptotic stabilization, and a secondary objective associated with the asymptotic stabilization of a reference orientation for a body-fixed frame. Beside provable stability and convergence properties in a large domain of operation, we believe that the conceptual simplicity of the solution, the non-requirement of switching between several control laws, and the ability to monitor smoothly transition phases (from fully-actuated mode to underactuated mode and vice versa) constitute valuable complementary assets. Its geometric nature shows through its construction in the framework of affine geometry and its expression, mostly coordinates-free, also distinguishes it from linear and other nonlinear control methods employed in the domains of aeronautics and aerial robotics.

The remainder of the paper is organized as follows. Section 2 explains modelling simplifications made at the actuation and control levels, and specifies the class of considered aerodynamic forces acting on the vehicle. Section 3 develops the control design in terms of primary and secondary objectives and shows how to complement feedback control laws derived in the fixed thrust direction case with a strategy capable of monitoring thrust tilting angle saturation efficiently. Section 4 reports simulation results which illustrate and validate the control approach on a tilted-quadrotor UAV. Section 5 briefly summarizes the contributions of the paper.

\section{PRELIMINARY MATERIAL}

\subsection{Notation}

The following notation is used throughout the paper.

- $E^{3}$ is the three-dimensional Euclidean affine space and $\boldsymbol{E}^{3}$ the associated Euclidean vector space (its direction), isomorphic to $\mathbb{R}^{3}$.

- The scalar product of two vectors $x$ and $y$ in $\mathbb{R}^{3}$ is denoted as $x \cdot y$, and their cross product as $x \times y$.

- Using the isomorphism between $\boldsymbol{E}^{3}$ and $\mathbb{R}^{3}$, similar operations can be defined on $\boldsymbol{E}^{3}$. The same notation, i.e. $\boldsymbol{x} \cdot \boldsymbol{y} \in \mathbb{R}$ and $\boldsymbol{x} \times \boldsymbol{y} \in \boldsymbol{E}^{3}$, is used for the scalar and cross products of two Euclidean vectors $\boldsymbol{x}, \boldsymbol{y}$.

- With $x \in \mathbb{R}^{n}, x^{T}$ stands for the transpose of $x$.

- The $i_{t h}$ component of a vector $x \in \mathbb{R}^{n}$ is denoted as $x_{i}$, and $\left\{e_{1}, \ldots, e_{n}\right\}$ is the canonical basis in $\mathbb{R}^{n}$.
- Given $x=\left(x_{1}, x_{2}, x_{3}\right)^{T} \in \mathbb{R}^{3}$, for the sake of conciseness $\left(x_{1} \boldsymbol{\imath}+x_{2} \boldsymbol{\jmath}+x_{3} \boldsymbol{k}\right)$ is written as $(\boldsymbol{\imath}, \boldsymbol{\jmath}, \boldsymbol{k}) x$.

- $G$ : vehicle's center of mass (CoM).

- $\mathcal{I}=\left\{O ; \boldsymbol{\iota}_{\mathbf{0}}, \boldsymbol{\jmath}_{\mathbf{0}}, \boldsymbol{k}_{\mathbf{0}}\right\}$ : inertial frame.

- $\mathcal{B}=\{G ; \boldsymbol{\imath}, \boldsymbol{\jmath}, \boldsymbol{k}\}$ : body-fixed frame.

- $\boldsymbol{u}$ : unit vector on the thrust axis.

- $\boldsymbol{\omega}_{\mathcal{I}}^{u}=\boldsymbol{u} \times \frac{d}{d t} \boldsymbol{u}_{\mid \mathcal{I}}$ : angular velocity of $\boldsymbol{u}$ with respect to the inertial frame.

- $\boldsymbol{\omega}_{\mathcal{B}}^{u}=\boldsymbol{u} \times \frac{d}{d t} \boldsymbol{u}_{\mid \mathcal{B}}$ : angular velocity of $\boldsymbol{u}$ with respect to the body-fixed frame.

- $\boldsymbol{\omega}=(\boldsymbol{\imath}, \boldsymbol{\jmath}, \boldsymbol{k}) \omega$ : angular velocity of the body-fixed frame w.r.t. the inertial frame.

- $u$ : vector of coordinates of $\boldsymbol{u}$ in the basis of $\mathcal{B}$, i.e. $\boldsymbol{u}=(\boldsymbol{\imath}, \boldsymbol{\jmath}, \boldsymbol{k}) u$.

- $\dot{u}$ : vector of coordinates of $\frac{d}{d t} \boldsymbol{u}_{\mid \mathcal{B}}$ in the basis of $\mathcal{B}$, i.e. $\frac{d}{d t} \boldsymbol{u}_{\mid \mathcal{B}}=(\boldsymbol{\imath}, \boldsymbol{\jmath}, \boldsymbol{k}) \dot{u}$.

- $m$ : vehicle's mass.

- $\boldsymbol{F}^{a}$ resultant of aerodynamic forces acting on the vehicle. - $\boldsymbol{T}=-T \boldsymbol{u}$ : thrust force, with the minus sign arising from a convention used for VTOL vehicles.

- $\boldsymbol{v}$ : CoM's velocity w.r.t. the inertial frame.

- $\boldsymbol{a}$ : CoM's acceleration w.r.t. the inertial frame.

- $\boldsymbol{g}$ : gravitational acceleration.

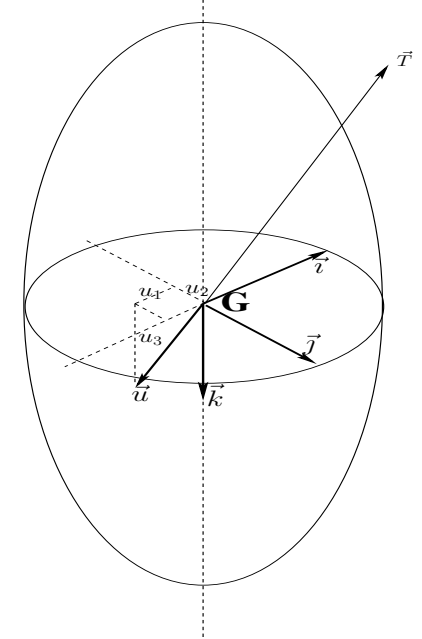

Fig. 1. Sketch representation of a VTOL vehicle with thrust tilting capability.

\subsection{Modelling Simplifications and Motion Equations}

In this section, a generic control model of a VTOL vehicle evolving in $3 \mathrm{D}$ space is proposed. The actuation inputs used to control the vehicle consist of i) a propulsion thrust $\boldsymbol{T}$ whose direction with respect to the vehicle's main body can be tilted, ii) a torque $\boldsymbol{\Gamma}$, independent of the thrust tilting actuation, used to modify the body's angular velocity $\boldsymbol{\omega}$ at will, and iii) torques needed to change the thrust direction. Nominally the resultant thrust force passes through the center of mass and thus exerts zero torque. Tilting the thrust direction may however create a parasitic torque $\boldsymbol{\Gamma}_{T}$. The control torque $\boldsymbol{\Gamma}$ then has to pre-compensate for this parasitic torque and also ensure that (almost) any desired angular velocity is physically 
obtained rapidly (instantaneously, in the ideal case). In practice, this torque is produced in various ways, typically by using propellers (e.g., VTOL vehicles Hamel et al. [2002], Ryll et al. [2012]), or rudders and flaps (e.g., aeroplanes Abzug and Larrabee [2002], ducted-fans Naldi et al. [2008]), or control moment gyros (e.g., spacecrafts Yoon and Tsiotras [2004]), etc. In order to give the reader an idea of how this torque can be calculated to ensure the swift convergence of $\boldsymbol{\omega}$ to a desired (timevarying) orientation velocity $\boldsymbol{\omega}^{\star}=(\boldsymbol{\imath}, \boldsymbol{\jmath}, \boldsymbol{k}) \omega^{\star}$, let us consider the classical Euler equation of the orientation dynamics expressed in the coordinates of body-fixed frame:

$$
I \dot{\omega}=-\omega \times I \omega+\Gamma_{e}+\Gamma
$$

with $I$ the vehicle's angular inertia matrix, $\Gamma_{e}$ the vector of coordinates in the body-fixed frame $\mathcal{B}$ of all "parasitic" torques (including $\Gamma_{T}$ and aerodynamic effects) acting on the vehicle. Then, calculating $\Gamma$ as follows

$$
\Gamma=-\Gamma_{e}+I \dot{\omega}^{\star}+\omega \times I \omega^{\star}-k_{\omega} I\left(\omega-\omega^{\star}\right) ; k_{\omega}>0
$$

and applying this torque to the vehicle yields the closedloop equation

$$
I \frac{d}{d t}\left(\omega-\omega^{\star}\right)=-\omega \times I\left(\omega-\omega^{\star}\right)-k_{\omega} I\left(\omega-\omega^{\star}\right)
$$

and hence the exponential stability of $\left(\omega-\omega^{\star}\right)=0$ with a rate of convergence of $\omega$ to $\omega^{\star}$ given by $k_{\omega}$. Under some extra assumptions upon $\omega^{\star}$ and $\Gamma_{e}$, one shows that a simple proportional feedback $\Gamma=-k_{\omega} I\left(\omega-\omega^{\star}\right)$ with $k_{\omega}>0$ large enough, suffices to render and maintain $\left|\omega-\omega^{\star}\right|$ smaller than any given small threshold.

The above considerations justify the conceptual "backstepping" assumption, commonly made, which consists in using the angular velocity $\boldsymbol{\omega}$, rather than the torque $\boldsymbol{\Gamma}$ which produces this velocity, as a control input. In the same order of idea, we will assume that the thrust intensity $T$, and the thrust-direction tilting angular velocity $\boldsymbol{\omega}_{\mathcal{B}}^{u}$, are the other control inputs at our disposal. The equations characterizing the system's dynamics are then

$$
\begin{aligned}
m \boldsymbol{a} & =m \boldsymbol{g}+\boldsymbol{F}^{a}-T \boldsymbol{u} \\
\frac{d}{d t}\{\boldsymbol{\imath}, \boldsymbol{\jmath}, \boldsymbol{k}\}_{\mid \mathcal{I}} & =\boldsymbol{\omega} \times\{\boldsymbol{\imath}, \boldsymbol{\jmath}, \boldsymbol{k}\} \\
\frac{d}{d t} \boldsymbol{u}_{\mid \mathcal{B}} & =\boldsymbol{\omega}_{\mathcal{B}}^{u} \times \boldsymbol{u}
\end{aligned}
$$

with $\boldsymbol{F}^{a}$ representing all forces, other than thrust and gravitation, applied to the vehicle's body. This vector is typically dominated by lift and drag aerodynamic forces whose intensities depend on the vehicle's longitudinal velocity (relatively to the ambient air) and, of particular importance for control purposes, on the vehicle's attitude when lift is not negligible. For the sake of simplification, and because the focus of the present paper is not to discuss control design aspects specifically related to the aerodynamic forces acting on the vehicle, we will here assume that $\boldsymbol{F}^{a}$ can be decomposed into the sum of two components as follows

$$
\boldsymbol{F}^{a}=\boldsymbol{F}_{1}^{a}+\boldsymbol{F}_{2}^{a}
$$

with $\boldsymbol{F}_{2}^{a}=F_{2}^{a} \boldsymbol{u}\left(F_{2}^{a} \in \mathbb{R}\right)$ and $\boldsymbol{F}_{1}^{a}$ a vector which, ideally, does not depend on the vehicle's orientation. For instance, in the case of a quadrotor UAV with tilted thrust-direction, alike the one considered in the simulation Section $4, \boldsymbol{F}^{a}$ involves a body-drag force $\boldsymbol{F}_{D}^{a}$ and an induced-drag force $\boldsymbol{F}_{I}^{a}$ generated by the airflow circulation around the rotors blades. Expressions of these forces are (Mahony et al. [2012]):

$$
\boldsymbol{F}_{D}^{a}=-c_{D}\left|\boldsymbol{v}^{a}\right| \boldsymbol{v}^{a}, \boldsymbol{F}_{I}^{a}=-c_{I} T\left(\boldsymbol{v}^{a}-\left(\boldsymbol{v}^{a} \cdot \boldsymbol{u}\right) \boldsymbol{u}\right)
$$

with $\boldsymbol{v}^{a}$ denoting the apparent air velocity, i.e. the vehicle's translational velocity minus the ambient air velocity, and $c_{D}$ and $c_{I}$ two aerodynamic coefficients. Summing up these two forces yields

$$
\begin{aligned}
\boldsymbol{F}^{a} & =\boldsymbol{F}_{D}^{a}+\boldsymbol{F}_{I}^{a} \\
& =\left(-c_{D}\left|\boldsymbol{v}^{a}\right| \boldsymbol{v}^{a}-c_{I} T \boldsymbol{v}^{a}\right)+\left(c_{I} T\left(\boldsymbol{v}^{a} \cdot \boldsymbol{u}\right) \boldsymbol{u}\right) \\
& =\boldsymbol{F}_{1}^{a}+\boldsymbol{F}_{2}^{a}
\end{aligned}
$$

with $\boldsymbol{F}_{1}^{a}:=-c_{D}\left|\boldsymbol{v}^{a}\right| \boldsymbol{v}^{a}-c_{I} T \boldsymbol{v}^{a}, \boldsymbol{F}_{2}^{a}:=c_{I} T\left(\boldsymbol{v}^{a} \cdot \boldsymbol{u}\right) \boldsymbol{u}$. Note that the direction of the drag component $\boldsymbol{F}_{1}^{a}$ is, by definition, aligned with the air velocity so that it does not depend on the vehicle's orientation. However, its amplitude depends on this orientation in the general case where the coefficients $c_{D}$ and $c_{I} T$ themselves vary with the vehicle's orientation. Nevertheless, there are flight domains where these coefficients can be considered as almost constant. For instance, in the absence of wind, for small vehicle's translational velocities the drag force is dominated by the airvelocity linearly dependent term $-c_{I} T \boldsymbol{v}^{a}$. Then, provided that the vehicle's translational acceleration intensity stays smaller than a certain threshold, the thrust $T$ essentially opposes the vehicle's weight $m g$ so that the drag force can, in this domain, be approximated by the orientation independent term $-c_{I} m g \boldsymbol{v}^{a}$ (assuming that the coefficient $c_{I}$ is itself almost constant). For the simulations of Section 4, this approximation is made at the control design level, whereas the "true" drag term $-c_{I} T \boldsymbol{v}^{a}$, with the control thrust $T$ depending on the the vehicle's orientation, is used to calculate the vehicle's motion.

In view of (5), the longitudinal dynamics equation (2) can be rewritten as

$$
m \boldsymbol{a}=m \boldsymbol{g}+\boldsymbol{F}_{1}^{a}-\bar{T} \boldsymbol{u}
$$

with $\bar{T}=T-F_{2}^{a}$ and an external force $\boldsymbol{F}_{1}^{a}$ which - in a certain flight domain and in the first approximation, as explained previously- does not depend on the vehicle's orientation.

\section{CONTROL DESIGN}

In the previous section we have justified the use of $\bar{T}, \boldsymbol{\omega}$, and $\boldsymbol{\omega}_{\mathcal{B}}^{u}$ as control inputs. The present section aims at working out feedback control expressions for these inputs.

\subsection{Primary Objective Realization}

Using the well known relations

$$
\frac{d}{d t} \boldsymbol{x}_{\mid \mathcal{I}}=\frac{d}{d t} \boldsymbol{x}_{\mid \mathcal{B}}+\boldsymbol{\omega} \times \boldsymbol{x}
$$

and

$$
\boldsymbol{x} \times(\boldsymbol{y} \times \boldsymbol{z})=(\boldsymbol{x} \cdot \boldsymbol{z}) \boldsymbol{y}-(\boldsymbol{x} \cdot \boldsymbol{y}) \boldsymbol{z}
$$

for any triplet $(\boldsymbol{x}, \boldsymbol{y}, \boldsymbol{z})$ of Euclidean vectors, and using the definitions of the angular velocities of $\boldsymbol{\omega}, \boldsymbol{\omega}_{\mathcal{I}}^{u}$, and $\boldsymbol{\omega}_{\mathcal{B}}^{u}$, it is simple to show that

$$
\boldsymbol{\omega}_{\mathcal{I}}^{u}=\boldsymbol{\omega}_{\mathcal{B}}^{u}+\boldsymbol{\omega}-(\boldsymbol{\omega} \cdot \boldsymbol{u}) \boldsymbol{u}
$$

Typically $\bar{T}$ and $\boldsymbol{\omega}_{\mathcal{I}}^{u}$ are determined in order to achieve a primary objective related to the vehicle's longitudinal motion. 
Reference velocity stabilization: For instance, consider the case where reference velocity asymptotic stabilization is the objective. Denoting the velocity error as $\tilde{\boldsymbol{v}}=\boldsymbol{v}-\boldsymbol{v}_{r}$, one obtains the following error equation

$$
\left.m \frac{d}{d t} \tilde{\boldsymbol{v}}\right|_{\mathcal{I}}=m\left(\boldsymbol{a}-\boldsymbol{a}_{r}\right)=-\bar{T} \boldsymbol{u}+\boldsymbol{F}
$$

with

$$
\boldsymbol{F}:=m \boldsymbol{g}+\boldsymbol{F}_{1}^{a}-m \boldsymbol{a}_{r}
$$

where $\boldsymbol{a}_{r}=\frac{d}{d t} \boldsymbol{v}_{r} \mid \mathcal{I}$ represents the acceleration of the reference trajectory w.r.t. the inertial frame.

Let $\bar{T}_{r}$ and $\boldsymbol{u}_{\boldsymbol{r}}$ denote the (positively signed) intensity and direction vector of $\boldsymbol{F}$ respectively, i.e.

$$
\left(\bar{T}_{r}, \boldsymbol{u}_{r}\right) \equiv\left(|\boldsymbol{F}|, \frac{\boldsymbol{F}}{|\boldsymbol{F}|}\right)
$$

As long as $|\boldsymbol{F}| \neq \mathbf{0}$, defining these terms poses no problem. We will assume from now on that this condition, which is automatically satisfied in the case of hovering thanks to the gravitational acceleration, is satisfied. As explained in Hua et al. [2009], the satisfaction of this condition is also necessary to the controllability of the linearized error system and the existence of conventional, either linear or nonlinear, control solutions. In view of (8), one must have $\bar{T} \boldsymbol{u}=\boldsymbol{F}$ at the equilibrium $\tilde{\boldsymbol{v}}=\mathbf{0}$. Therefore, $\bar{T}$ must converge to $\bar{T}_{r}$, whereas the thrust direction $\boldsymbol{u}$ must converge to $\boldsymbol{u}_{r}$

The above considered problem of asymptotic stabilization of a reference velocity has already received solutions (see Hua et al. [2009] or Hua et al. [2013]) which, modulo minor modifications and transposition details, yield the following control expressions

$$
\begin{aligned}
\bar{T} & =\bar{T}_{r} \boldsymbol{u} \cdot \boldsymbol{u}_{r}+k_{1} m \boldsymbol{u} \cdot \tilde{\boldsymbol{v}} \\
\boldsymbol{\omega}_{\mathcal{I}}^{u} & =\frac{k_{2} m}{\bar{T}_{r}} \boldsymbol{u} \times \tilde{\boldsymbol{v}}+\frac{\left(k_{3}+\bar{k}_{3}\right)}{\left(1+\boldsymbol{u} \cdot \boldsymbol{u}_{r}\right)^{2}} \boldsymbol{u} \times \boldsymbol{u}_{r} \\
& +\left(\boldsymbol{\omega}_{\mathcal{I}}^{u_{r}}-\left(\boldsymbol{\omega}_{\mathcal{I}}^{u_{r}} \cdot \boldsymbol{u}\right) \boldsymbol{u}\right)
\end{aligned}
$$

with $\boldsymbol{\omega}_{\mathcal{I}}^{u_{r}}:=\boldsymbol{u}_{r} \times \frac{d}{d t} \boldsymbol{u}_{r \mid \mathcal{I}}$ the instantaneous angular velocity of $\boldsymbol{F}, k_{1,2,3}$ positive gains, and $\bar{k}_{3}=2 \dot{\bar{T}}_{r}\left(1+\boldsymbol{u} \cdot \boldsymbol{u}_{r}\right) / \bar{T}_{r}$. This controller is derived by considering the following (positive) Lyapunov function candidate:

$$
\mathcal{L}=\frac{1}{k_{2}} \frac{\bar{T}_{r}^{2}}{m^{2}}\left(1-\boldsymbol{u} \cdot \boldsymbol{u}_{r}\right)+\frac{1}{2}|\tilde{\boldsymbol{v}}|^{2}
$$

whose time-derivative along any solution to the controlled system is (see the proof in the Appendix A):

$$
\dot{\mathcal{L}}=-\frac{k_{3}}{k_{2}} \frac{\bar{T}_{r}^{2}}{m^{2}} \frac{\left|\boldsymbol{u} \times \boldsymbol{u}_{r}\right|^{2}}{\left(1+\boldsymbol{u} \cdot \boldsymbol{u}_{r}\right)^{2}}-k_{1}(\boldsymbol{u} \cdot \tilde{\boldsymbol{v}})^{2}(\leq 0)
$$

Provided that $|\boldsymbol{F}| \neq \mathbf{0}$, that the reference velocity and acceleration are bounded, and that $\boldsymbol{u}+\boldsymbol{u}_{r} \neq \mathbf{0}$ initially, then $\left(\tilde{\boldsymbol{v}}=\mathbf{0}, \boldsymbol{u}-\boldsymbol{u}_{r}=\mathbf{0}\right)$ is asymptotically stable (see complementary details in Hua et al. [2009]). Control robustification when $\boldsymbol{F}$ or $\boldsymbol{u}+\boldsymbol{u}_{r}$ vanishes is discussed in Hua et al. [2009].

Reference position stabilization: If, instead of reference velocity stabilization, the primary objective is the tracking of a reference position trajectory, the same control can be used modulo minor modifications involving (bounded) integrals of velocity and position errors. The Lyapunov function candidate used for convergence and stability analysis is modified accordingly Hua et al. [2009], Hua et al. [2013].
For the sake of precision, let $\boldsymbol{x}:=\boldsymbol{O} \boldsymbol{G}$ and $\boldsymbol{x}_{\boldsymbol{r}}$ respectively denote the vehicle's position and reference position w.r.t an inertial frame, and let $\tilde{\boldsymbol{x}}:=\boldsymbol{x}-\boldsymbol{x}_{r}$ denote the position error vector. The above mentioned modifications consist, for instance, in replacing the definition (10) of $\bar{T}_{r}$ and $u_{r}$ by

$$
\left(\bar{T}_{r}, \boldsymbol{u}_{r}\right) \equiv\left(\left|\boldsymbol{F}_{\xi}\right|, \frac{\boldsymbol{F}_{\xi}}{\left|\boldsymbol{F}_{\xi}\right|}\right)
$$

where:

- $\boldsymbol{F}_{\xi}:=m \boldsymbol{g}+\boldsymbol{F}_{1}^{a}-m \boldsymbol{a}_{r}+m k_{I} \frac{d^{2}}{d t^{2}} \boldsymbol{z}_{\mid \mathcal{I}}+m \boldsymbol{\sigma}(\tilde{\boldsymbol{\xi}})$

- $\tilde{\boldsymbol{\xi}}:=\tilde{\boldsymbol{x}}+k_{I} \boldsymbol{z} ; \quad k_{I}>0$

- $\boldsymbol{z}$ is a bounded integral of the position error $\tilde{\boldsymbol{x}}$ defined as the solution to the following equation

$$
\frac{d^{2}}{d t^{2}} \boldsymbol{z}_{\mid \mathcal{I}}=-k_{\dot{z}} \frac{d}{d t} \boldsymbol{z}_{\mid \mathcal{I}}+\mathbf{s a t}^{\frac{\ddot{z}_{\max }}{2}}\left(k_{z}\left(-\boldsymbol{z}+\mathbf{s a t}^{\Delta_{z}}\left(\boldsymbol{z}+\frac{\tilde{\boldsymbol{x}}}{k_{z}}\right)\right)\right)
$$

with zero initial conditions, i.e. $\boldsymbol{z}(0)=\frac{d}{d t} \boldsymbol{z}_{\mid \mathcal{I}}(0)=\mathbf{0}$, positive numbers $k_{z}, k_{\dot{z}}, \ddot{z}_{\max }$ and $\Delta_{z}$, and sat ${ }^{\Delta}$ denoting the classical saturation function defined by $\operatorname{sat}^{\Delta}(\boldsymbol{x})=$ $\min (1, \Delta /|\boldsymbol{x}|) \boldsymbol{x}$.

- $\boldsymbol{\sigma}$ is a bounded function satisfying some properties (detailed in Hua et al. [2009] and Hua et al. [2013]) and an example of which is the function defined by $\boldsymbol{\sigma}(\boldsymbol{y}):=$ $\beta\left(\beta^{2}|\boldsymbol{y}|^{2} / \eta^{2}+1\right)^{-\frac{1}{2}} \boldsymbol{y}$, with $\beta$ and $\eta$ denoting positive numbers.

The last modification concerns the term $\tilde{\boldsymbol{v}}$ which, in the control expressions (11) and (12), has to be replaced by $\tilde{\boldsymbol{v}}_{\xi}:=\tilde{\boldsymbol{v}}+k_{I} \frac{d}{d t} \boldsymbol{z}_{\mid \mathcal{I}}$.

\subsection{Secondary Objective Realization}

The thrust intensity $T$ (or equivalently, $\bar{T}$ ) has been determined previously (the relation (11)). It thus remains to determine $\boldsymbol{\omega}$ and $\boldsymbol{\omega}_{\mathcal{B}}^{u}$ that satisfy (7), as imposed by the realization of the primary objective, and also allow for the realization of a secondary objective.

Let $\boldsymbol{\omega}^{\star}$ denote the angular velocity control that would be used for the secondary control objective if the thrust tilting angle was not limited. For instance, this objective can be the asymptotic stabilization of the body-fixed frame vector $\boldsymbol{k}$ at a reference time-varying unit vector $\boldsymbol{k}_{r}$. Then, a possible control, whose expression is obtained in the same way as the control $\boldsymbol{\omega}_{\mathcal{I}}^{u}$ in (12) by rendering the timederivative of the Lyapunov function candidate $\left(1-\boldsymbol{k} \cdot \boldsymbol{k}_{r}\right)$ negative when $\boldsymbol{k} \neq \boldsymbol{k}_{r}$, is

$$
\boldsymbol{\omega}^{\star}=\frac{k_{4}}{\left(1+\boldsymbol{k} \cdot \boldsymbol{k}_{r}\right)^{2}} \boldsymbol{k} \times \boldsymbol{k}_{r}+\lambda \boldsymbol{k}+\left(\boldsymbol{\omega}_{\mathcal{I}}^{k_{r}}-\left(\boldsymbol{\omega}_{\mathcal{I}}^{k_{r}} \cdot \boldsymbol{k}\right) \boldsymbol{k}\right)
$$

with $k_{4}>0, \lambda \in \mathbb{R}$ and $\boldsymbol{\omega}_{\mathcal{I}}^{k_{r}}:=\boldsymbol{k}_{r} \times \frac{d}{d t} \boldsymbol{k}_{r \mid \mathcal{I}}$. The indetermination of $\lambda$ corresponds to the unused d.o.f associated with rotations about the $\boldsymbol{k}$ direction. If the secondary objective is to asymptotically stabilize a reference orientation for the vehicle's body, then a possible control is

$$
\boldsymbol{\omega}^{\star}=-k_{4} \tan (\theta / 2) \boldsymbol{\nu}+\boldsymbol{\omega}_{r} ; \quad k_{4}>0
$$

with $\theta \boldsymbol{\nu}$ the rotation vector associated with the orientation error between the body-fixed frame and the reference orientation, and $\boldsymbol{\omega}_{r}$ the reference angular velocity associated with the reference orientation.

Let us define

$$
\boldsymbol{\omega}_{\mathcal{B}}^{u \star}:=\boldsymbol{\omega}_{\mathcal{I}}^{u}-\left(\boldsymbol{\omega}^{\star}-\left(\boldsymbol{\omega}^{\star} \cdot \boldsymbol{u}\right) \boldsymbol{u}\right)
$$


Since $\boldsymbol{\omega}_{\mathcal{B}}^{u \star}$ satisfies (7) when $\boldsymbol{\omega}$ is equal to the unconstrained solution $\boldsymbol{\omega}^{\star}$, it is the thrust tilting angular velocity that would be used in the unconstrained case to achieve both primary and secondary objectives. However, due to the thrust tilting angle limitation, modified expressions for $\boldsymbol{\omega}$ and $\boldsymbol{\omega}_{\mathcal{B}}^{u}$ have to be worked out. To this aim, let us first specify the maximum value of the thrust tilting angle and set

$$
\delta=\max \left(\sqrt{u_{1}^{2}+u_{2}^{2}}\right)
$$

so that the maximum tilting angle is equal to $\arcsin (\delta)$ $\in\left[0, \frac{\pi}{2}\right)$.

Define

$$
\dot{\boldsymbol{u}}^{\star}:=\boldsymbol{\omega}_{\mathcal{B}}^{u \star} \times \boldsymbol{u}
$$

and denote $\dot{u}^{\star}$ as the vector of coordinates of $\dot{\boldsymbol{u}}^{\star}$ in the basis of the body-fixed frame $\mathcal{B}$, i.e. $\dot{\boldsymbol{u}}^{\star}=\{\boldsymbol{\imath}, \boldsymbol{\jmath}, \boldsymbol{k}\} \dot{u}^{\star}$. Using $x_{1,2}$ to denote the vector of first two components of $x \in \mathbb{R}^{3}$, we propose to modify the tilting angle according to the following control law:

$$
\dot{u}_{1,2}=-k_{u} u_{1,2}+k_{u} \operatorname{sat}^{\delta}\left(u_{1,2}+\frac{\dot{u}_{1,2}^{\star}}{k_{u}}\right)
$$

with $k_{u}$ a positive number (not necessarily constant), and $\operatorname{sat}^{\delta}($.$) the classical saturation function defined by$ $\operatorname{sat}^{\delta}(x)=\min (1, \delta /|x|) x$, with $x \in \mathbb{R}^{2}$ in the present case. Then, $\boldsymbol{\omega}_{\mathcal{B}}^{u}$ is given by

$$
\boldsymbol{\omega}_{\mathcal{B}}^{u}=\boldsymbol{u} \times \frac{d}{d t} \boldsymbol{u}_{\mid \mathcal{B}}
$$

with $\frac{d}{d t} \boldsymbol{u}_{\mid \mathcal{B}}=\{\boldsymbol{\imath}, \boldsymbol{J}, \boldsymbol{k}\} \dot{u}$ and the third component of $\dot{u}$ given by $\dot{u}_{3}=-\frac{u_{1,2}^{T} \dot{u}_{1,2}}{u_{3}}=-\frac{u_{1,2}^{T} \dot{u}_{1,2}}{\sqrt{1-u_{1}^{2}-u_{2}^{2}}}$, using the fact that $\boldsymbol{u}$ is a unit vector. One easily verifies from (20) and (21) that $\dot{u}=\dot{u}^{\star}$ whenever $\left|u_{1,2}+\dot{u}_{1,2}^{\star} / k_{u}\right| \leq \delta$. This indicates that, for $u_{1,2}$ to be dominant in the left-hand side of this inequality, $k_{u}$ should be chosen large enough. In view of (21), one also has the relations

$$
\begin{aligned}
\frac{1}{2} \frac{d}{d t}\left|u_{1,2}\right|^{2} & =-k_{u}\left|u_{1,2}\right|^{2}+k_{u} u_{1,2}^{T} \mathrm{sat}^{\delta}\left(u_{1,2}+\dot{u}_{1,2}^{\star} / k_{u}\right) \\
& \leq-k_{u}\left|u_{1,2}\right|^{2}+k_{u} \delta\left|u_{1,2}\right|
\end{aligned}
$$

from which one deduces that $\left|u_{1,2}\right|$ remains smaller or equal to $\delta$, provided that the initial value of $\left|u_{1,2}\right|$ is itself chosen smaller or equal to $\delta$. Therefore, this control law i) respects the imposed limitation on the thrust tilting angle, and ii) allows for the realization of the secondary objective when $\left|u_{1,2}+\dot{u}_{1,2}^{\star} / k_{u}\right| \leq \delta$.

It then remains to determine $\boldsymbol{\omega}$. An obvious choice is

$$
\boldsymbol{\omega}=\boldsymbol{\omega}_{\mathcal{I}}^{u}-\boldsymbol{\omega}_{\mathcal{B}}^{u}+\left(\boldsymbol{\omega}^{\star} \cdot \boldsymbol{u}\right) \boldsymbol{u}
$$

Indeed, one deduces from this relation that $\boldsymbol{\omega} \cdot \boldsymbol{u}=\boldsymbol{\omega}^{\star} \cdot \boldsymbol{u}$ and, subsequently, that $\boldsymbol{\omega}_{\mathcal{B}}^{u}=\boldsymbol{\omega}_{\mathcal{B}}^{u \star}$ and $\boldsymbol{\omega}=\boldsymbol{\omega}^{\star}$ when $\left|u_{1,2}+\dot{u}_{1,2}^{\star} / k_{u}\right| \leq \delta$. Moreover, the equality (7) is always satisfied with this choice, in accordance with the priority given to the realization of the primary objective.

Remark 1. Thrust-tilting may involve only a single rotation about a body-fixed axis (see, e.g., Cetinsoy et al. [2012], Notarstefano and Hauser [2010], Russo et al. [2011], Papachristos et al. [2011]) by contrast with the two d.o.f. rotation case here considered. A straightforward adaptation of the proposed control design for the realization of the secondary objective then consists in replacing the control law (21) by

$$
\left\{\begin{array}{l}
\dot{u}_{1}=-k_{u} u_{1}+k_{u} \operatorname{sat}^{\delta}\left(u_{1}+\dot{u}_{1}^{\star} / k_{u}\right) \\
\dot{u}_{2}=u_{2}=0
\end{array}\right.
$$

\section{APPLICATION TO A THRUST-TILTED QUADROTOR UAV}

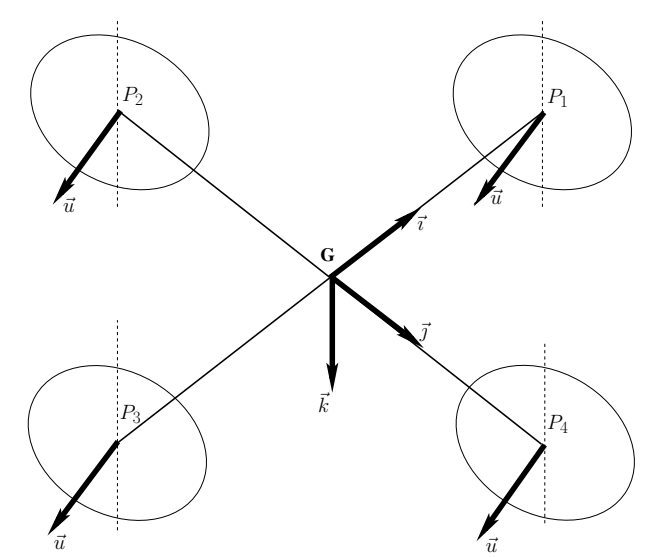

Fig. 2. Sketch representation of a quadrotor with thrust tilting capability.

The above control solution has been tested on the model of a quadrotor UAV sketched on Fig. 2. We assume that the four rotor axes can be simultaneously tilted and in the same direction corresponding to the overall thrust direction $\boldsymbol{u}$. Let $P_{i}(i=1, \ldots, 4)$ be the pivoting points of the four rotors, with their positions in the body frame defined by

$$
\begin{array}{lll}
\boldsymbol{G} \boldsymbol{P}_{1}=h \boldsymbol{k}+d \boldsymbol{\imath}, & \boldsymbol{G} \boldsymbol{P}_{2}=h \boldsymbol{k}-d \boldsymbol{\jmath} \\
\boldsymbol{G} \boldsymbol{P}_{3}=h \boldsymbol{k}-d \boldsymbol{\imath}, & \boldsymbol{G} \boldsymbol{P}_{4}=h \boldsymbol{k}+d \boldsymbol{\jmath}
\end{array}
$$

with $h \in \mathbb{R}$ and $d>0$. On Fig. $2, h=0$. Let $\varpi_{i}$ $(i=1, \ldots, 4)$ denote the angular velocities of the four rotors. According to Hamel et al. [2002], the i-th rotor generates a thrust force $\boldsymbol{T}_{i}=\mu \varpi_{i}^{2} \boldsymbol{u}$ and a drag torque $\boldsymbol{Q}_{i}=\lambda_{i} \kappa \varpi_{i}^{2} \boldsymbol{u}$, with $\mu$ and $\kappa$ two aerodynamic coefficients and $\lambda_{i}=1$ (resp. -1 ) if $i$ is odd (resp. even). The thrust $\boldsymbol{T}$ and the torque vector $\boldsymbol{\Gamma}=(\boldsymbol{\imath}, \boldsymbol{\jmath}, \boldsymbol{k}) \Gamma$ generated by the four rotors are thus given by

$$
\begin{aligned}
& \boldsymbol{T}= \sum_{i} \boldsymbol{T}_{i}=\mu\left(\sum_{i} \varpi_{i}^{2}\right) \boldsymbol{u} \\
& \boldsymbol{\Gamma}=\sum_{i}\left(\boldsymbol{G P}_{i} \times \boldsymbol{T}_{i}+\boldsymbol{Q}_{i}\right) \\
&=d \mu\left(\varpi_{1}^{2}-\varpi_{3}^{2}\right)(\boldsymbol{\imath} \times \boldsymbol{u})-d \mu\left(\varpi_{2}^{2}-\varpi_{4}^{2}\right)(\boldsymbol{\jmath} \times \boldsymbol{u}) \\
& \quad+h \mu \sum_{i} \varpi_{i}^{2}(\boldsymbol{k} \times \boldsymbol{u})+\sum_{i} \lambda_{i} \kappa \varpi_{i}^{2} \boldsymbol{u}
\end{aligned}
$$

One deduces the following relation between the vector of rotors angular velocities $\varpi_{i}$ on the one hand, and the vector composed of the thrust intensity and the control torque components on the other hand:

$$
\left[\begin{array}{c}
T \\
\Gamma
\end{array}\right]=A_{m o t}\left[\begin{array}{l}
\varpi_{1}^{2} \\
\varpi_{2}^{2} \\
\varpi_{3}^{2} \\
\varpi_{4}^{2}
\end{array}\right]
$$

with the allocation matrix $A_{m o t}=\left[\begin{array}{llll}a_{c 1} & a_{c 2} & a_{c 3} & a_{c 4}\end{array}\right]$, 


$$
\begin{gathered}
a_{c 1}:=\left[\begin{array}{c}
\mu \\
-h \mu u_{2}+\kappa u_{1} \\
h \mu u_{1}-d \mu u_{3}+\kappa u_{2} \\
d \mu u_{2}+\kappa u_{3}
\end{array}\right], a_{c 2}:=\left[\begin{array}{c}
\mu \\
-h \mu u_{2}-d \mu u_{3}-\kappa u_{1} \\
h \mu u_{1}-\kappa u_{2} \\
d \mu u_{1}-\kappa u_{3}
\end{array}\right] \\
a_{c 3}:=\left[\begin{array}{c}
\mu \\
-h \mu u_{2}+\kappa u_{1} \\
h \mu u_{1}+d \mu u_{3}+\kappa u_{2} \\
-d \mu u_{2}+\kappa u_{3}
\end{array}\right], a_{c 4}:=\left[\begin{array}{c}
\mu \\
-h \mu u_{2}+d \mu u_{3}-\kappa u_{1} \\
h \mu u_{1}-\kappa u_{2} \\
-d \mu u_{1}-\kappa u_{3}
\end{array}\right]
\end{gathered}
$$

Since $A_{m o t}$ is invertible $\left(\operatorname{det}\left(A_{m o t}\right)=8 \kappa d^{2} \mu^{3} u_{3}>0\right), T$ and $\Gamma$ can be given any desired values -modulo the constraint of positivity of the rotors angular velocities and the limited range of velocities imposed by power limitations of the rotors- and can thus be used as independent control variables. The direct application of the proposed control strategy relies on this actuation property.

\subsection{Simulation results}

Specifications of the simulated vehicle are given in Tab. 1.

Table 1. Specifications of the simulated UAV.

\begin{tabular}{|c||c|}
\hline Specification & Numerical Value \\
\hline Mass $m[\mathrm{~kg}]$ & 1.5 \\
Moment of Inertia $I\left[\mathrm{~kg} \mathrm{~m}^{2}\right]$ & $\operatorname{diag}(0.028,0.028,0.06)$ \\
Level arm values $[h, d][\mathrm{m}]$ & {$[0.05,0.2]$} \\
Thrust angle limitation $[\mathrm{rad}]$ & $\pi / 6$ \\
Body drag coefficient $c_{D}\left[\mathrm{~kg} \mathrm{~m}^{-1}\right]$ & 0.0092 \\
Induced drag coefficient $c_{I}\left[\mathrm{~kg} \mathrm{~s}^{-1}\right]$ & 0.025 \\
\hline
\end{tabular}

Concerning the calculation of the torque $\Gamma$ in charge of producing the desired body angular velocity defined by (22), we have used $\Gamma=-k_{\omega} I\left(\omega-\omega^{\star}\right)+\omega \times I \omega^{\star}$, with $\omega$ denoting the vehicle's angular velocity obtained by integration of the Euler equation (1), $\omega^{\star}$ the vector of coordinates, expressed in the body-fixed frame $\mathcal{B}$, of the reference angular velocity defined by (22), and $k_{\omega}$ a positive gain. Due to the parasitic torque induced by the chosen non-zero value of $h$ (one of the parameters characterizing the position of the propellers) and the non precompensation of both this torque and the reference angular acceleration $\dot{\omega}^{\star}$ in the expression of $\Gamma$, there remains a residual error between $\omega$ and $\omega^{\star}$ in the general case. We have made this simplification in the control calculation in order to test the robustness of the proposed control design against (inevitable) modelling errors. This explains residual position tracking errors that are observable in the reported simulation results.

The primary objective considered for these simulations is the position tracking of an eight-shaped Lissajous trajectory defined by

$$
\boldsymbol{x}_{r}=5 \sin \left(a_{r} t\right) \boldsymbol{\imath}_{0}+5 \sin \left(2 a_{r} t\right) \boldsymbol{J}_{0}
$$

By changing the parameter $a_{r}$ one modifies the time period of a complete run, as well as the associated reference velocity and acceleration. Two values of $a_{r}(2 \pi / 15$ and $\pi / 5)$ are considered. The first one corresponds to a "slow" run (Simulation 1) that involves non-saturated thrustdirection tilt angles, whereas the second one corresponds to a "fast" run (Simulation 2) along portions of which the tilt angle attains its maximum value.

The chosen secondary objective is the stabilization of the vehicle's attitude about the identity matrix. In particular, the realization of this objective requires the vehicle's

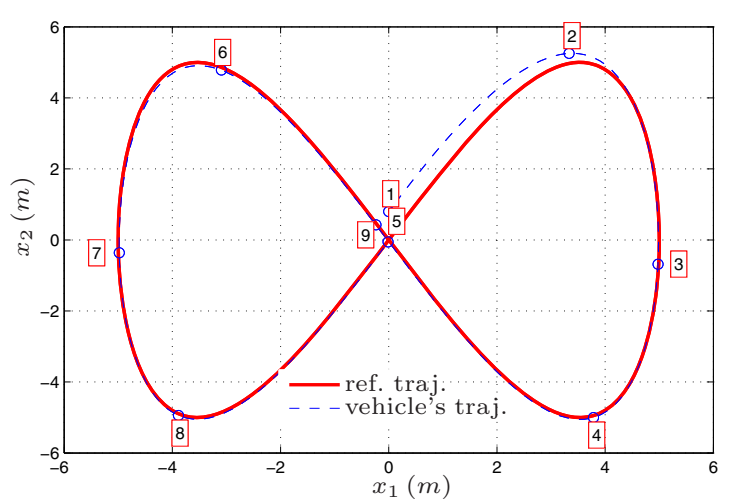

Fig. 3. Reference and vehicle trajectories projected on the horizontal plane (Simulation 1).

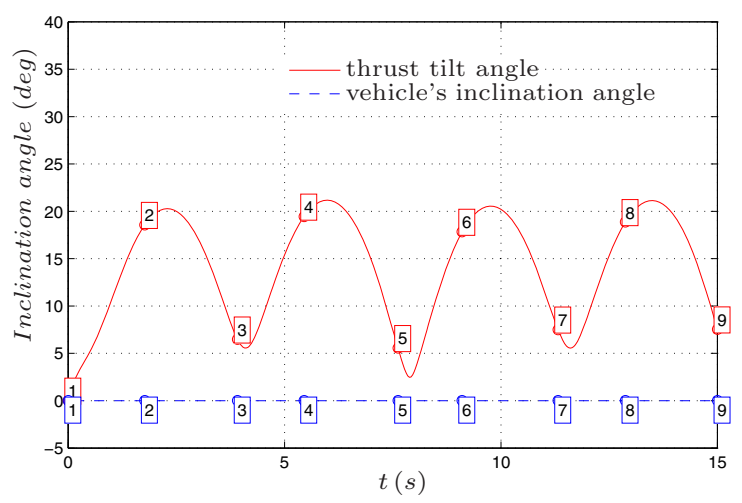

Fig. 4. Thrust and vehicle inclination angles vs. time (Simulation 1).

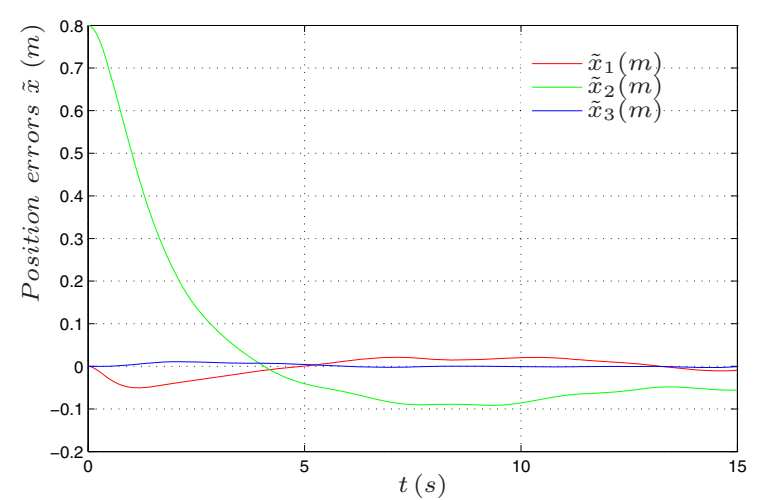

Fig. 5. Position tracking errors vs. time (Simulation 1).

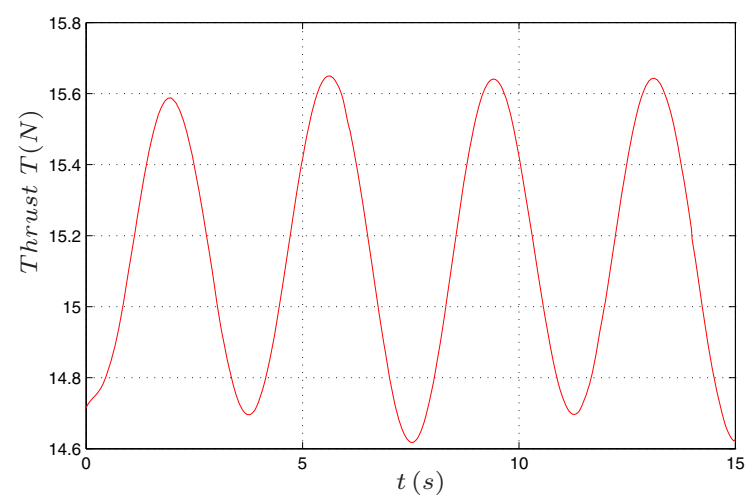

Fig. 6. Thrust intensity vs. time (Simulation 1).

plane containing the rotors' pivot points $P_{1,2,3,4}$ to remain horizontal all the time. The associated angular velocity control $\boldsymbol{\omega}^{\star}$ is given by (17). 
The control gains and other parameters involved in the calculation of the control inputs are chosen as follows:

- $k_{1}=2, k_{2}=7.56, k_{3}=19.2, k_{I}=0.4$,

- $\beta=0.81, \eta=6$,

- $k_{\dot{z}}=4, k_{z}=4, \Delta_{z}=1, \ddot{z}_{\max }=0.5$,

- $k_{4}=10, k_{u}=20$,

- $k_{\omega}=20$.

In the control expression, the aerodynamic force $\boldsymbol{F}_{1}^{a}$ is approximated by $\boldsymbol{F}_{1}^{a} \approx-c_{D}\left|\boldsymbol{v}_{r}\right| \boldsymbol{v}_{r}-c_{I} m g \boldsymbol{v}_{r}$ which is obtained by replacing $T$ and $\boldsymbol{v}^{a}$ by $m g$ and $\boldsymbol{v}_{r}$, respectively. The time-derivative of this force, also needed for the control calculation, is calculated accordingly. Initial conditions for the vehicle's configuration are as follows:

$$
\left\{\begin{array}{l}
\boldsymbol{x}(0)=0.8 \boldsymbol{J}_{0}, \quad \boldsymbol{v}(0)=5 a_{r} \boldsymbol{\imath}_{0}+10 a_{r} \boldsymbol{J}_{0}, \\
\{\boldsymbol{\imath}(0), \boldsymbol{\jmath}(0), \boldsymbol{k}(0)\}=\left\{\boldsymbol{\iota}_{0}, \boldsymbol{J}_{0}, \boldsymbol{k}_{0}\right\} \\
\boldsymbol{u}(0)=\boldsymbol{k}(0), \quad \boldsymbol{\omega}(0)=\boldsymbol{\omega}_{\mathcal{B}}^{u}(0)=\mathbf{0} .
\end{array}\right.
$$

- Simulation $1\left(a_{r}=2 \pi / 15\right)$ : The time period for a complete run is $15 \mathrm{~s}$. The projection on the horizontal plane of the path followed by the vehicle's CoM is shown in Fig. 3. Variations w.r.t. time of the vehicle and thrust inclination angles, of the position tracking errors, and of the thrust magnitude, are shown in Figs. 4-6. In Figs. 3 and 4 the nine highlighted points correspond to time-instants when the reference trajectory involves large acceleration variations. From Fig. 3 one can observe that the vehicle catches up with, and subsequently closely follows, the reference trajectory. Despite a rather aggressive reference trajectory, with an average longitudinal velocity of about $4 \mathrm{~m} / \mathrm{s}$ and accelerations sometimes exceeding $3 \mathrm{~m} / \mathrm{s}^{2}$, the vehicle's base remains always horizontal (see Fig. 4). One can also observe from Fig. 4 that the thrust-direction tilt angle never reaches its maximum value (equal to $\pi / 6 \mathrm{rad}$ ). Both goals are thus achieved (almost) perfectly in this case.

- Simulation $2\left(a_{r}=\pi / 5\right)$ : This simulation is devised to illustrate the effects of thrust tilting saturation and the corresponding control monitoring. The time-period for a complete run is reduced to $10 \mathrm{~s}$ and the reference trajectory is more aggressive than the one in Simulation 1. Results are shown in Figs. 7-11. One can now observe from Fig. 8 that the vehicle's inclination periodically departs from zero when the thrust-direction tilt angle attains its maximum value. While the primary position tracking task is again performed perfectly, the secondary objective is, as expected, imperfectly realized in this case. However, the body's inclination returns to the desired zero value as soon as the thrust tilt angle needed to achieve the secondary objective re-enters the domain of allowed tilt angles, a behaviour that we find satisfactory.

\section{CONCLUSION}

Nonlinear control of VTOL vehicles endowed with thrust tilting capability has been addressed and a generic control solution exploiting thrust-tilting augmentation has been devised. The proposed solution potentially applies to a large panel of aerial vehicles with extended flight envelopes. It involves a primary objective consisting in the asymptotic stabilization of either a reference velocity or a reference position trajectory, and a secondary objective consisting in the asymptotic stabilization of either a reference direction for one of the body-base vectors or a

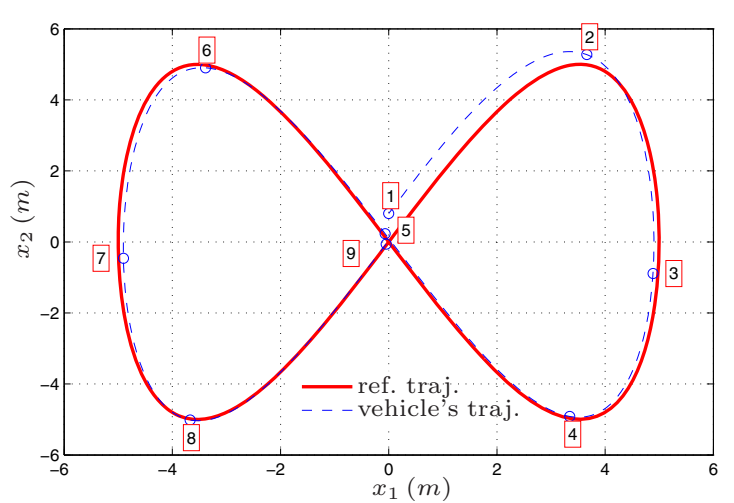

Fig. 7. Reference and vehicle trajectories projected on the horizontal plane (Simulation 2).

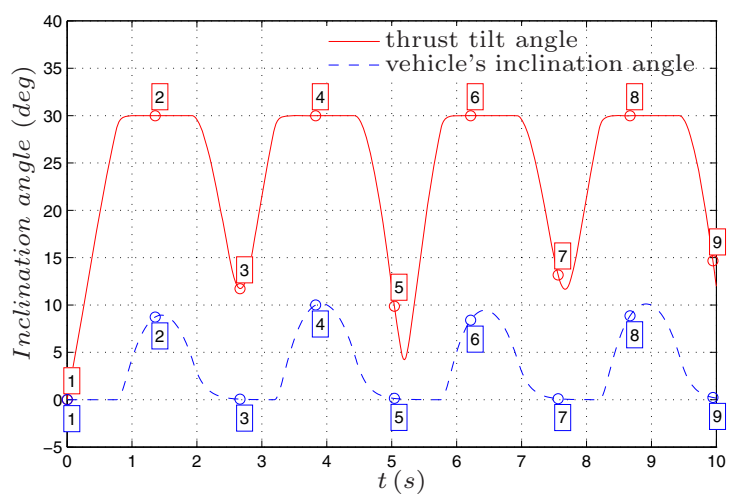

Fig. 8. Thrust and vehicle inclination angles vs. time (Simulation 2).

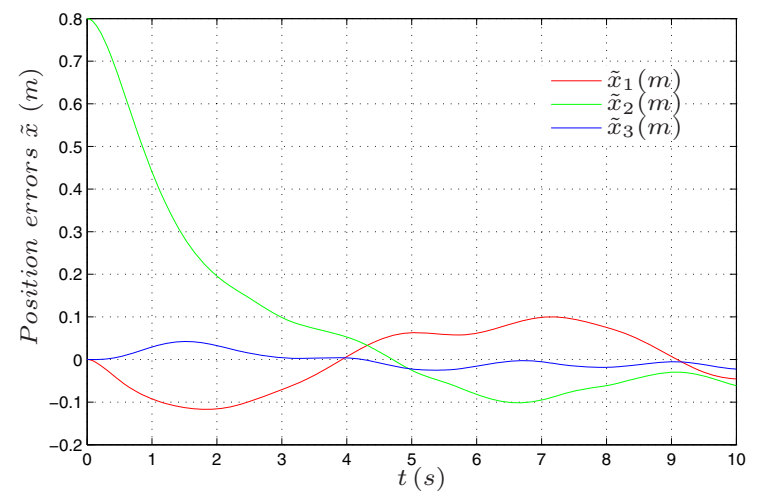

Fig. 9. Position tracking errors vs. time (Simulation 2).

complete reference orientation for the body-fixed frame. A major original outcome of the present study is the definition of a control solution that takes thrust-tilting limitations into account explicitly. We view it also as new contribution to the ongoing development of a unified nonlinear approach to the control of aerial vehicles. The proposed thrust tilting control strategy has so far been validated only in simulation. Experiments carried out on physical devices are thus needed to adapt the approach to the specificities of each flying device and assert its practical usefulness.

\section{REFERENCES}

M.J. Abzug and E.E. Larrabee. Airplane Stability and Control. Cambridge University Press, second edition, 2002. 


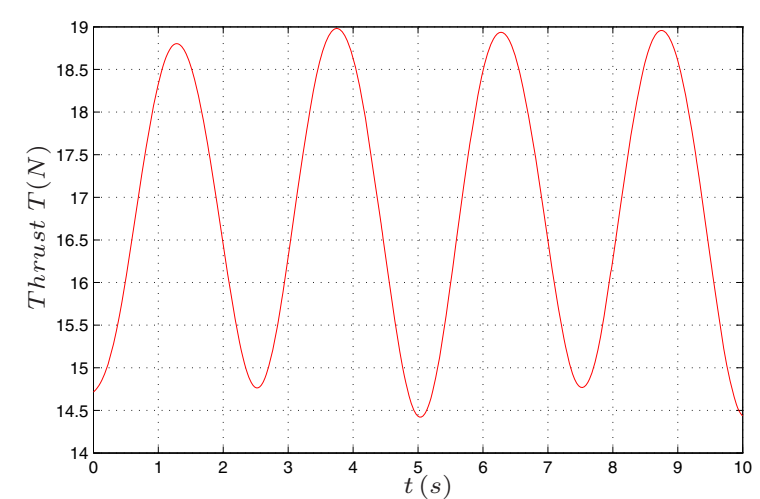

Fig. 10. Thrust intensity vs. time (Simulation 2).

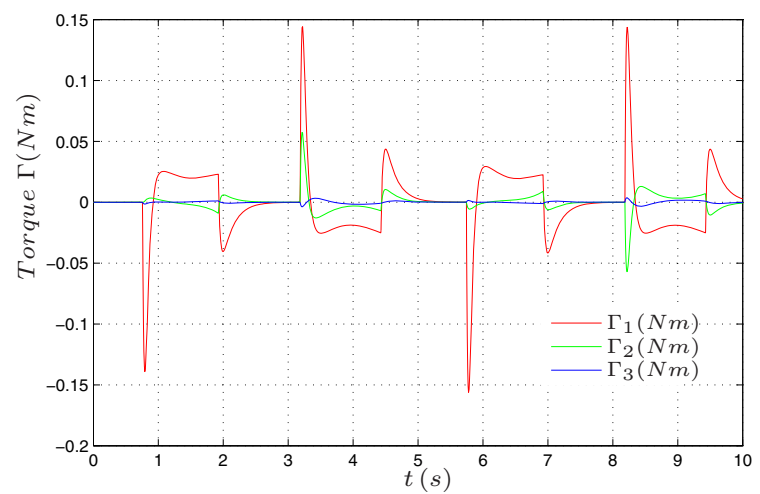

Fig. 11. Torque inputs vs. time (Simulation 2).

E. Cetinsoy, S. Dikyar, C. Hancer, K.T. Oner, E. Sirimoglu, M. Unel, and M.F. Aksit. Design and construction of a novel quad tilt-wing uav. Mechatronics, 22(6): 723-745, 2012.

T. Hamel, R. Mahony, R. Lozano, and J. Ostrowski. Dynamic modelling and configuration stabilization for an X4-flyer. In IFAC World Congress, pages 200-212, 2002.

M.-D. Hua, T. Hamel, P. Morin, and C. Samson. A control approach for thrust-propelled underactuated vehicles and its application to VTOL drones. IEEE Transactions on Automatic Control, 54(8):1837-1853, 2009.

M.-D. Hua, T. Hamel, P. Morin, and C. Samson. Introduction to Feedback Control of Underactuated VTOL Vehicles: A review of basic control design ideas and principles. IEEE Control Systems Magazine, 33(1):6175, 2013.

F. Kendoul, I. Fantoni, and R. Lozano. Modeling and control of a small autonomous aircraft having two tilting rotors. In IEEE Conference on Decision and Control, pages 8144-8149, 2005.

R. Mahony, V. Kumar, and P. Corke. Multirotor Aerial Vehicles: Modeling, Estimation, and Control of Quadrotor. IEEE Robotics \&S Automation Magazine, 19(3):20$32,2012$.

R. Naldi, L. Marconi, and A. Sala. Modelling and Control of a Miniature Ducted-Fan in Fast Forward Flight. In American Control Conference, pages 2552-2557, 2008.

G. Notarstefano and J. Hauser. Modeling and dynamics exploration of a tilt-rotor VTOL aircraft. In IFAC Symposium on Nonlinear Control Systems, pages 119124, 2010.
C. Papachristos, K. Alexis, and A. Tzes. Design and experimental attitude control of an unmanned tilt-rotor aerial vehicle. In IEEE International Conference on Advanced Robotics, pages 465-470, 2011.

J.-M. Pflimlin, P. Binetti, P. Souères, T. Hamel, and D. Trouchet. Modeling and attitude control analysis of a ducted-fan micro aerial vehicle. Control Engineering Practice, pages 209-218, 2010.

E. Russo, G. Notarstefano, and J. Hauser. Dynamics exploration and aggressive maneuvering of a longitudinal vectored thrust VTOL aircraft. In IEEE Conference on Decision and Control, pages 119-124, 2011.

M. Ryll, H.H. Bulthoff, and P.R. Giordano. Modeling and Control of a Quadrotor UAV with Tilting Propellers. In IEEE International Conference on Robotics and Automation, pages 4606-4613, 2012.

S. Salazar-Cruz, F. Kendoul, R. Lozano, and I. Fantoni. Real-time stabilization of a small three-rotor aircraft. IEEE Transactions on Aerospace and Electronic Systems, 44(2):783-794, 2008.

H. Yoon and P. Tsiotras. Singularity analysis of variablespeed control moment gyros. AIAA Journal of Guidance, Control, and Dynamics, 27(3):374-386, 2004.

\section{Appendix A. TIME-DERIVATIVE OF THE} LYAPUNOV FUNCTION (13)

One has

$$
\begin{aligned}
\frac{d}{d t} & \left(1-\boldsymbol{u} \cdot \boldsymbol{u}_{r}\right)=-\frac{d}{d t} \boldsymbol{u}_{\mid \mathcal{I}} \cdot \boldsymbol{u}_{r}-\boldsymbol{u} \cdot \frac{d}{d t} \boldsymbol{u}_{r \mid \mathcal{I}} \\
& =-\left(\boldsymbol{\omega}_{\mathcal{I}}^{u} \times \boldsymbol{u}\right) \cdot \boldsymbol{u}_{r}-\boldsymbol{u} \cdot\left(\boldsymbol{\omega}_{\mathcal{I}}^{u_{r}} \times \boldsymbol{u}_{r}\right) \\
& =\left(\boldsymbol{u}_{r} \times \boldsymbol{u}\right) \cdot\left(\boldsymbol{\omega}_{\mathcal{I}}^{u}-\boldsymbol{\omega}_{\mathcal{I}}^{u_{r}}\right)
\end{aligned}
$$

so that

$$
\begin{aligned}
& \frac{d}{d t} \frac{\bar{T}_{r}^{2}}{k_{2} m}\left(1-\boldsymbol{u} \cdot \boldsymbol{u}_{r}\right) \\
& =\frac{\bar{T}_{r}^{2}}{k_{2} m}\left(\left(\boldsymbol{u}_{r} \times \boldsymbol{u}\right) \cdot\left(\boldsymbol{\omega}_{\mathcal{I}}^{u}-\boldsymbol{\omega}_{\mathcal{I}}^{u_{r}}\right)+\frac{2 \overline{\bar{T}}_{r}}{\bar{T}_{r}}\left(1-\boldsymbol{u} \cdot \boldsymbol{u}_{r}\right)\right) \\
& =\frac{\bar{T}_{r}^{2}}{k_{2} m}\left(\boldsymbol{u}_{r} \times \boldsymbol{u}\right) \cdot\left(\boldsymbol{\omega}_{\mathcal{I}}^{u}-\boldsymbol{\omega}_{\mathcal{I}}^{u_{r}}+\frac{\bar{k}_{3}}{\left(1+\boldsymbol{u} \cdot \boldsymbol{u}_{r}\right)^{2}}\left(\boldsymbol{u}_{r} \times \boldsymbol{u}\right)\right)
\end{aligned}
$$

where we have used the expression of $\bar{k}_{3}$ and the identity $1-\left(\boldsymbol{u} \cdot \boldsymbol{u}_{r}\right)^{2}=\left|\boldsymbol{u}_{r} \times \boldsymbol{u}\right|^{2}$. From (8) one has also

$$
\begin{aligned}
& \frac{d}{d t} \frac{1}{2}|\tilde{\boldsymbol{v}}|^{2}=\frac{1}{m} \tilde{\boldsymbol{v}} \cdot(-\bar{T} \boldsymbol{u}+\boldsymbol{F}) \\
& =\frac{1}{m}\left(-\bar{T} \tilde{\boldsymbol{v}} \cdot \boldsymbol{u}+\bar{T}_{r} \tilde{\boldsymbol{v}} \cdot \boldsymbol{u}_{r}\right) \\
& =\frac{1}{m}\left(\bar{T}_{r} \boldsymbol{u} \cdot \boldsymbol{u}_{r}-\bar{T}\right) \tilde{\boldsymbol{v}} \cdot \boldsymbol{u}+\frac{\bar{T}_{r}}{m}\left(\boldsymbol{u}_{r} \times \boldsymbol{u}\right) \cdot(\tilde{\boldsymbol{v}} \times \boldsymbol{u})
\end{aligned}
$$

where we have used the identity $\boldsymbol{u}_{r}=\boldsymbol{u} \times\left(\boldsymbol{u}_{r} \times \boldsymbol{u}\right)+$ $\left(\boldsymbol{u} \cdot \boldsymbol{u}_{r}\right) \boldsymbol{u}$. The time-derivative of the candidate Lyapunov function (13) is the sum of the left-hand side terms of (A.1) and (A.2). Therefore

$$
\begin{aligned}
& \frac{d}{d t} \mathcal{L}=\frac{1}{m}\left(\bar{T}_{r} \boldsymbol{u} \cdot \boldsymbol{u}_{r}-\bar{T}\right) \tilde{\boldsymbol{v}}+\frac{\bar{T}_{r}^{2}}{k_{2} m}\left(\boldsymbol{u}_{r} \times \boldsymbol{u}\right) . \\
& \quad\left(\boldsymbol{\omega}_{\mathcal{I}}^{u}-\boldsymbol{\omega}_{\mathcal{I}}^{u_{r}}+\frac{\bar{k}_{3}}{\left(1+\boldsymbol{u} \cdot \boldsymbol{u}_{r}\right)^{2}}\left(\boldsymbol{u}_{r} \times \boldsymbol{u}\right)+\frac{k_{2} m}{\bar{T}_{r}} \tilde{\boldsymbol{v}} \times \boldsymbol{u}\right)
\end{aligned}
$$

It then suffices to replace $\bar{T}$ and $\boldsymbol{\omega}_{\mathcal{I}}^{u}$ by their expressions (11) and (12) in the right-hand side of the above equality to obtain (14). 